\title{
$\angle$ Research Square \\ Efficacy of Isolated Bacteriophage Against Biofilm Embedded Colistin-Resistant Acinetobacter baumannii
}

\section{Saeedeh Ebrahimi}

Isfahan University of Medical Sciences

Behnam Sisakhtpour

Isfahan University of Medical Sciences

Arezoo Mirzaei

Isfahan University of Medical Sciences

Vajihe Karbasizadeh

Isfahan University of Medical Sciences

Sharareh Moghim ( $\nabla$ moghim@med.mui.ac.ir)

\section{Research note}

Keywords: Colistin-resistant Acinetobacter baumannii, Biofilm, Bacteriophage, Phage therapy

Posted Date: April 20th, 2020

DOI: https://doi.org/10.21203/rs.3.rs-19395/v1

License: (c) (1) This work is licensed under a Creative Commons Attribution 4.0 International License.

Read Full License

Version of Record: A version of this preprint was published at Gene Reports on March 1st, 2021. See the published version at https://doi.org/10.1016/j.genrep.2020.100984. 


\section{Abstract}

Objective: Acinetobacter baumannii is responsible for most nosocomial infections in hospitals. It has the ability to form biofilms and has a high degree of antibiotic resistance. Colistin is one of the last therapeutic options for the treatment of Multi Drug Resistance infections. Recently, strains of this pathogen resistance to the colistin were reported increasingly. Therefore, alternative antibacterial methods such as phage therapy are being researched.

Results: From 15 MDR A. baumannii clinical isolates, $26.6 \%$ were resistant to colistin, $80 \%$ were able to produce strong biofilm, and $20 \%$ produce weak biofilm. The isolated lytic phage (IsfAB78) was able to reduce the biofilm by up to $87 \%$. Since most of the MDR colistin-resistant strains produce biofilm, and MDR A. baumannii infections are difficult to treat, development of phage therapy could be an alternative in the future. Phage IsfAB78 is a good candidate for this purpose.

\section{Introduction}

Acinetobacter baumannii, as a gram-negative opportunistic pathogen, is classified amongst the most dangerous multi-drug resistance (MDR) pathogens, known as ESKAPE (Enterococcus faecium, Staphylococcus aureus, Klebsiella pneumoniae, Acinetobacter baumannii, Pseudomonas aeruginosa and species of Enterobacter) [1]. This opportunistic pathogen is associated with major outbreaks of nosocomial infections especially in intensive care units (ICUs) [2-4]. It produces various types of infections such as pneumonia, bacteremia, and meningitis [5]. These bacteria possess many resistance mechanisms, including the drug-degrading enzymes, infringing the infiltration and different efflux pumps [6]. Currently, the only resistant antibiotics against MDR A. baumannii are Tigecycline, Monocycline, Polymyxins, and Colistin [7]. One of the most important virulence features of this bacteria is the ability to produce biofilms and high adaptability to survive in harsh environmental conditions such as hospitals. Biofilms are a complex community of microbes that are adhered to biotic or abiotic surfaces [8]. They often enclosed by thick polysaccharides which decrease the accessibility of antibiotics and the immune system to the bacterial community and make them hard to exclude [9]. Moreover, antibiotic resistance causes the bacteria to spread which may increase the incidence of nosocomial infections caused by bacteria [10]. In addition, more A. baumannii strains were found to be resistant to all known antibiotics, which has urged to find an alternative approach to treat MDR bacteria [11]. In recent decades, increasing evidence has shown the possibility of phage therapy to treat drug-resistant bacterial infections [12]. Bacteriophages are present in all the environments containing their host and play an important role in biological activities [13]. Phages have bacteriolytic performance and can kill bacterial cells in biofilms [14]. The most important characteristics of phages include their specificity and proliferation within the host and at the site of infection, with no side effects [15].

In this study, we found out the biofilm formation of clinical isolates of colistin-resistant. Acinetobacter baumannii, and assessed their sensitivity to the isolated bacteriophage. 


\section{Main Text}

\section{Collection and identification of isolates}

In this study, all 15 A. baumannii clinical samples, were collected from patients with burn wound infections at Isfahan Medical University hospitals during the years 2017-2018. All A. baumannii isolates were confirmed by conventional phenotypic and molecular methods [16].

\section{Determining the Minimum Inhibitory Concentration}

Minimum Inhibitory Concentration (MIC) for Colistin (Sigma-Aldrich, USA) was performed using the standard microdilution broth technique according to CLSI guidelines (Clinical and Laboratory Standards Institute). The MIC was defined as the lowest concentration of antibiotic that inhibits the growth of bacteria after overnight incubation. The test was repeated three times for each isolate. E. coli (ATCC 25922) was used as a positive control[11].

\section{Biofilm formation}

Acinetobacter baumannii biofilm formation was carried out in 96-well flat-bottomed polystyrene tissue culture plate (SPL, Korea). Isolates were cultured for 48 hours in $5 \mathrm{ml}$ tryptic soy broth (TSB) supplemented with $1 \%$ glucose. The cultures were diluted to $1: 1000$ (equivalent to $10^{8} \mathrm{CFU}$ ), and $200 \mu \mathrm{L}$ of each aliquot were added to each well and incubated at $37^{\circ} \mathrm{C}$. After $24 \mathrm{~h}$ of incubation, the plates were rinsed three times thoroughly with normal saline (NS) and allowed to dry at room temperature for 1 hour. Finally, they were stained with crystal violet (1\%) and their optical density was measured at $570 \mathrm{~nm}$. The test was repeated three times $[17,18]$. Biofilm formation was classified into 4 categories according to the amount of optical density: (1) strong $\left(4 O D_{\text {control }}<O D_{\text {test }}\right)$; (2) medium biofilm producer $\left(2 O D_{c}<O D_{t} 4 O D_{c}\right)$; (3) weak biofilm producer $\left(O D_{c}<O D_{t} \geq 2 O D_{c}\right)$; and (4) non-biofilm producer $\left(O D_{t} \geq O D_{c}\right)(22)$. Finally, three colistin-resistant MDR-AB isolates, with strong biofilms, were selected and used in experiments.

\section{Phage Isolation}

IsfAB78 is a lytic phage isolated from hospital wastewater. The characteristics of this phage are described in our previous study (23). Briefly, $50 \mathrm{ml}$ of water samples from hospital wastewater were centrifuged at 13,000 rpm for $10 \mathrm{~min}$. The supernatant was filtered through a $0.45 \mu \mathrm{m}$ pore size membrane and mixed with $50 \mathrm{ml}$ of $2 x$ nutrient broth (containing $\mathrm{MgSO}_{4}, 1 \mathrm{mM} \mathrm{CaCl}_{2}, 1 \mathrm{mM}$ ) and $1 \mathrm{ml}$ $\left(10^{8} \mathrm{CFU}\right)$ of a 24 -hour culture of the indicator bacteria. After $24 \mathrm{~h}$ incubation at $35^{\circ} \mathrm{C}$ and $160 \mathrm{rpm}$, a few drops of chloroform were added, and 15 minutes later the mixture was centrifuged for 10 minutes at $4000 \mathrm{~g}$. The supernatant was filtered through a 0.45 pore filter membrane. The double-layer agar and spot test methods are used to detect phage plaque formation [19]. 


\section{Double-Layer Agar}

Melted nutrient agar medium (1.15\%) was poured on a plate, and after solidification, $1 \mathrm{ml}$ of the filtered phages were mixed with $1 \mathrm{ml}$ of 24 -hour bacterial culture $\left(10^{8} \mathrm{CFU}\right)$ and added to liquid nutrient agar medium $(0.7 \%)$ with the temperature of $45^{\circ} \mathrm{C}$, and the mixture was placed on the plate containing nutrient agar. The solidified plate was incubated for 24 hours at $37^{\circ} \mathrm{C}$. The clear plaques were picked by sterile loops and inoculated into $5 \mathrm{ml}$ of nutrient broth supplemented with fresh cultured bacterial suspension. After $24 \mathrm{~h}$ at $35^{\circ} \mathrm{C}$ and $160 \mathrm{rpm}$, the mixture was centrifuged at $4000 \mathrm{~g}$ for $15 \mathrm{~min}$ and the supernatant was filtered through a $0.2 \mu \mathrm{m}$ syringe filter. The filtrate was processed through double-layer assay as described above and again an individual plaque was picked. This step was repeated several times in order to increase the purity of the phage $[19,20]$. The titer of the isolated phage was determined by standard plaque assay and stored at $4^{\circ} \mathrm{C}$ for further use [21].

\section{Spot Test}

Briefly, $1 \mathrm{ml}$ of bacterial culture at the concentration of $10^{8} \mathrm{pfu} / \mathrm{ml}$ was inoculated in $2.5 \mathrm{ml}$ of the melted nutrient agar medium $(0.07 \%)$ and the mixture was poured onto a plate containing $1.5 \%$ nutrient agar, where two-layer plates were created. When the top layer was solidified, $10 \mu \mathrm{l}$ of the filtered phage were spotted in the plate and incubated at $37^{\circ} \mathrm{C}$ for 24 hours. The created plaque indicates bacterial susceptibility to the phage [22].

\section{Optimum phage eradication concentration}

The antibacterial activity of the isolated phage in the biofilm was measured using different concentrations of the phage. Optimum phage eradication concentration indicates the best concentration of the phage leading to clear wells in 96-well culture plates. For this experiment biofilm was grown according to the described method. After the biofilm formation, the planktonic cells were discarded and each well was washed 3 times with normal saline (NS). A total of $3 \times 10^{9} \mathrm{PFU}, 3 \times 10^{8} \mathrm{PFU}, 3 \times 10^{7} \mathrm{PFU}, 3 \times$ $10^{6} \mathrm{PFU}, 3 \times 10^{5} \mathrm{PFU}$ equal to $\mathrm{MOI}$ of $10,1,0.1,0.01,0.001$ in a total volume of $200 \mu \mathrm{l}$ of the diluted phage were added to each well. The plate was incubated for 48 hours at $37^{\circ} \mathrm{C}$. The well-containing biofilms and not added phage were used as a negative control, and wells containing the medium were used as sterility control.

\section{Evaluation of antibacterial of the phage activity against biofilm}

Finally, MTT assay (3- [4.5-dimethylthiazol-2-yl] -2.5-diphenyltetrazolium bromide) was used to determine the biofilm formation [23]. For this purpose, bacterial suspension was removed and $50 \mu \mathrm{l}$ of MTT (0.3\%) in 
$150 \mu$ l of PBS was added for 2 hours at $37^{\circ} \mathrm{C}$. Afterward, the MTT solution was removed and $150 \mu \mathrm{DMSO}$ lysis solution was added into each well and kept at room temperature and in a dark environment for 30 minutes. The absorbance of the solution was measured at a wavelength of $550 \mathrm{~nm}$ using a Microplate Reader Model 550 (BioRad). In order to measure the individual biofilm formation of each isolate, the ratio of the biofilm OD of the isolate that was incubated with the phage was calculated in relation to the biofilm OD of the same isolate without phage (native biofilm).

\section{Results}

\section{Minimum inhibitory concentration}

The MIC of the isolates against the colistin antibiotic was done according to CLSI. From 15 identified MDR A. baumannii isolates, $26.6 \%$ showed strong resistance (MIC of $\leq 4$ ) to the colistin, and $73.3 \%$ isolates were susceptible (MIC $\geq 2$ ). E. coli 25922 was used as positive control (Table1).

Table 1

MIC (Minimum Inhibitory Concentration) all isolates were determined according CLSI and 73.3 percentage were sensitive about colistin.

\begin{tabular}{|c|c|c|c|c|c|c|}
\hline \multirow[t]{2}{*}{$\begin{array}{l}\text { Antimicrobial } \\
\text { agent }\end{array}$} & \multicolumn{3}{|c|}{$\begin{array}{l}\text { ANTIMICROBIAL RESISTANT } \\
\text { LEVEL }\end{array}$} & \multicolumn{3}{|c|}{$\begin{array}{l}\text { MINIMUM INHIBITORY CONCENTRATION } \\
\text { (MIC) }\end{array}$} \\
\hline & $S$ & I & $\mathbf{R}$ & $S$ & I & $\mathbf{R}$ \\
\hline COLISTIN & $\% 73 / 3$ & - & $\% 26 / 6$ & $\leq 2$ & 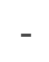 & $\geq 4$ \\
\hline
\end{tabular}

\section{Biofilm formation}

The results of biofilm formation in the microtiter plate showed that $20 \%$ of isolates formed weak biofilm, $40 \%$ isolates created intermediate biofilm, and $40 \%$ produced strong biofilm (Fig. 1).

\section{Phage Identification}

Phage IsfAB78 was isolated from hospital wastewater (23). MDR-AB biofilm used as a host indicator for the isolation of lytic phages. As shown in Fig. 2, the phage can form large clear plaques with a $13 \mathrm{~mm}$ diameter on the bacterial lawns, indicating lytic property of the phage. IsfAB78 has an approximately $100 \mathrm{~nm}$ long, six-sided symmetry and can be classified as Myoviridae. The phage was tested for their infectivity to all $15 \mathrm{~A}$. baumannii clinical isolates in order to determine their host range and lytic potential. Three strong producer colistin-resistant MDR A. baumannii clinical isolates were chosen for further study. Phage IsfAB78 can significantly cause lysis in an MDR A. baumannii culture after 40 minutes.

\section{Effect of Phage on Biofilm of Different Isolates}


Three colistin-resistant MDR A. baumannii isolates were selected to assess the potential of the phage to disintegrate biofilms. The established biofilm was treated with different concentrations of phages. The results were represented as a percentage of the biofilm biomass in control samples that were left untreated. The lytic phage seemed to be able to reduce the biofilm (19-87\%) but the percentage of biofilm reduction was dependent on the concentration of added phages. At low concentration $\left(10^{6}\right.$ $\mathrm{pfu} / \mathrm{ml}$ ), the reduction in biofilm was significant (up to over $87 \%$ ) (Fig. 3 (supplementary)).

\section{Discussion}

Treatment of bacterial infections involving biofilm formation is very difficult, especially for multidrugresistant pathogens. Therefore, there is an urgent need to develop new alternative treatments to combat such increasing infections. As a nosocomial pathogen, the multi-drug resistant Acinetobacter baumannii (MDR-AB) illustrates an increasing global health threat [24]. Colistin is now considered as the last resort treatment for gram-negative bacilli including MDR- A. baumannii. Unfortunately, the increasing use of colistin has resulted in the emergence of resistance as well [22-25]. Therefore, looking for an alternative therapeutic method is imperative. Bacteriophage therapy may provide new treatment strategies to combat drug-resistant bacterial infections associated with biofilm. Phages have demonstrated to damage biofilm by disintegrating its structural components [26].

In this study, a specific lytic phage was isolated from hospital wastewater. In similar studies, lytic bacteriophages are isolated on different bacteria, and also investigate their effects and properties on the considered bacteria [27, 28]. The resistance of 15 Acinetobacter baumannii was also assessed against different antibiotics. All isolates were multi-drug resistant. The results of the antibiotic susceptibility tests were in agreement with other studies in the region including Sadegifard et al. and Sepahvand et al. Also, the result of MIC of colistin in the same region indicates that colistin resistance is increasing during the last years which is an alarming issue that must be considered. This study, in accordance with other works in the region, showed that antimicrobial resistance of $A$. baumannii in Iran is increasing[29,30]. Biofilm production was initially assessed on the isolates. In this study $80 \%$ of the isolates produce biofilm, and the rest were weak biofilm producers. In another study, it was shown that $60 \%$ and $62 \%$ of their isolates produced biofilm [31,32]. The percentages of the biofilm-forming bacteria obtained in our study were slightly more than other researchers because of lower number of the experimented samples. Although the number of samples is effective in the experiment, the increase in pathogenicity of the bacteria cannot be denied. The effect of the isolated phage was evaluated on three biofilm embedded colistin-resistant MDR$A B$ isolates using the MTT assay. The analysis of the data showed that the isolated lytic phage could reduce the biofilm content of these isolates up to $87 \%$. Bacteriophages have significant applications to degrade biofilm on medical devices' surface. P. mirabilis and $E$. coli could reduce about a $90 \%$ reduction in biofilm when treated with phage as compared to untreated [33]. For all isolates the results were not the same; The phage did not result in total removal of some isolates biofilms. It seems for more effective and complete removal of biofilms, a combination of more than one lytic phage or combination of phage with antibiotics may be useful. The results also showed that different phage dilutions affect the removal of 
biofilm. The best dilution in all three isolates was $\mathrm{MOI} 0.01\left(0^{6} \mathrm{pfu} / \mathrm{ml}\right)$. Similar to other studies, this study showed that the isolated lytic phage had an impact on the biofilm of Acinetobacter baumannii [20, 22, 23, 34]. The use of phage has been proposed as an alternative method due to the high drug resistance of Acinetobacter baumannii and bacterial adaptation at hospital levels. Due to the appropriate performance of phage in reducing the biofilm, the phage is suggested as a therapeutic agent for Acinetobacter baumannii. However, for the required therapeutic usage, further studies are needed on animal models, genome identification and its properties, and also on the extent of phage co-administration with antibiotics.

\section{Limitations}

The limitations that we confront with that in this study were the lack of several phages to investigate the effect of them on isolates, and also in-vivo and cell culture analysis should do in further investigation.

\section{Abbreviations}

MDR: multi-drug resistant

AB: Acinetobacter baumannii

MTT: 3- [4.5-dimethylthiazol-2-yl] -2.5-diphenyltetrazolium bromide

DMSO: Dimethyl sulfoxide

Mol: multiplicity of infection

OD: optical density

PBS: Phosphate buffered saline

\section{Declarations}

\section{Ethics approval and consent to participate}

Not applicable.

\section{Consent for publication}

Not applicable.

\section{Availability of data and materials}

The datasets used and/or analyzed during the current study are available from the corresponding author on reasonable request 
Competing interests

The authors declare that they have no competing interests.

Funding

This study was financially supported by Isfahan University of Medical Sciences, Isfahan, I.R. Iran through the Grant No 397306.

\section{Authors' contributions}

SE was a significant contributor to doing and writing the manuscript.BS and AM and VK collaborated in doing the thesis that results in the paper.SM Designed and supervised the manuscript. All authors read and approved the final manuscript.

\section{Acknowledgements}

This study was financially supported by Isfahan University of Medical Sciences, Isfahan, I.R. Iran through the Grant No 397306.

\section{References}

1. Yaneja $\mathrm{N}$, Kaur H: Insights into newer antimicrobial agents against Gram-negative bacteria. Microbiology insights 2016, 9:MBI. S29459.

2. Chen L-K, Kuo S-C, Chang K-C, Cheng C-C, Yu P-Y, Chang C-H, Chen T-Y, Tseng C-C: Clinical Antibioticresistant Acinetobacter baumannii strains with higher susceptibility to environmental phages than antibiotic-sensitive strains. Scientific reports 2017, 7(1):6319.

3. Mihu MR, Martinez LR: Novel therapies for treatment of multi-drug resistant Acinetobacter baumannii skin infections. Virulence 2011, 2(2):97-102.

4. Howard A, O'Donoghue M, Feeney A, Sleator RD: Acinetobacter baumannii: an emerging opportunistic pathogen. Virulence 2012, 3(3):243-250.

5. Xie R, Zhang XD, Zhao Q, Peng B, Zheng J: Analysis of global prevalence of antibiotic resistance in Acinetobacter baumannii infections disclosed a faster increase in OECD countries. Emerging microbes \& infections 2018, 7(1):1-10.

6. Peleg AY, Seifert $H$, Paterson DL: Acinetobacter baumannii: emergence of a successful pathogen. Clinical microbiology reviews 2008, 21(3):538-582.

7. Yang C-H, Su P-W, Moi S-H, Chuang L-Y: Biofilm Formation in Acinetobacter Baumannii: GenotypePhenotype Correlation. Molecules 2019, 24(10):1849.

8. Lee C-R, Lee JH, Park M, Park KS, Bae IK, Kim YB, Cha C-J, Jeong BC, Lee SH: Biology of Acinetobacter baumannii: pathogenesis, antibiotic resistance mechanisms, and prospective treatment options. Frontiers in cellular and infection microbiology 2017, 7:55. 
9. Hakyemez IN, Kucukbayrak A, Tas T, Yikilgan AB, Akkaya A, Yasayacak A, Akdeniz H: Nosocomial Acinetobacter baumannii infections and changing antibiotic resistance. Pakistan journal of medical sciences 2013, 29(5):1245.

10. Leung CYJ, Weitz JS: Modeling the synergistic elimination of bacteria by phage and the innate immune system. Journal of theoretical biology 2017, 429:241-252.

11. Thummeepak R, Kitti T, Kunthalert D, Sitthisak S: Enhanced antibacterial activity of acinetobacter baumannii bacteriophage øABP-01 endolysin (LysABP-01) in combination with colistin. Frontiers in microbiology 2016, 7:1402.

12. García-Quintanilla M, Pulido MR, López-Rojas R, Pachón J, McConnell MJ: Emerging therapies for multidrug resistant Acinetobacter baumannii. Trends in microbiology 2013, 21(3):157-163.

13. Kakasis A, Panitsa G: Bacteriophage therapy as an alternative treatment for human infections. A comprehensive review. International journal of antimicrobial agents 2019, 53(1):16-21.

14. Sulakvelidze A, Alavidze Z, Morris JG: Bacteriophage therapy. Antimicrobial agents and chemotherapy 2001, 45(3):649-659.

15. Viertel TM, Ritter K, Horz H-P: Viruses versus bacteria-novel approaches to phage therapy as a tool against multidrug-resistant pathogens. Journal of Antimicrobial Chemotherapy 2014, 69(9):23262336.

16. Asif M, Alvi IA, Rehman SU: Insight into Acinetobacter baumannii: pathogenesis, global resistance, mechanisms of resistance, treatment options, and alternative modalities. Infection and drug resistance 2018, 11:1249.

17. Longo F, Vuotto C, Donelli G: Biofilm formation in Acinetobacter baumannii. New Microbio/ 2014, 37(2):119-127.

18. Wu W-S, Chen C-C, Chuang Y-C, Su B-A, Chiu Y-H, Hsu H-J, Ko W-C, Tang H-J: Efficacy of combination oral antimicrobial agents against biofilm-embedded methicillin-resistant Staphylococcus aureus. Journal of Microbiology, Immunology and Infection 2013, 46(2):89-95.

19. Ghajavand H, Esfahani BN, Havaei A, Fazeli H, Jafari R, Moghim S: Isolation of bacteriophages against multidrug resistant Acinetobacter baumannii. Research in pharmaceutical sciences 2017, 12(5):373.

20. Yele AB, Thawal ND, Sahu PK, Chopade BA: Novel lytic bacteriophage AB7-IBB1 of Acinetobacter baumannii: isolation, characterization and its effect on biofilm. Archives of virology 2012, 157(8):1441-1450.

21. Mirzaei MK, Nilsson AS: Isolation of phages for phage therapy: a comparison of spot tests and efficiency of plating analyses for determination of host range and efficacy. PLOS One 2015, 10(3): $\mathrm{e} 0118557$.

22. Kusradze I, Karumidze N, Rigvava S, Dvalidze T, Katsitadze M, Amiranashvili I, Goderdzishvili M: Characterization and testing the efficiency of Acinetobacter baumannii phage vB-GEC_Ab-M-G7 as an antibacterial agent. Frontiers in microbiology 2016, 7:1590. 
23. Kwiatek M, Parasion S, Rutyna P, Mizak L, Gryko R, Niemcewicz M, Olender A, Łobocka M: Isolation of bacteriophages and their application to control Pseudomonas aeruginosa in planktonic and biofilm models. Research in microbiology 2017, 168(3):194-207.

24. Gunn JS, Bakaletz LO, Wozniak DJ: What's on the outside matters: the role of the extracellular polymeric substance of Gram-negative biofilms in evading host immunity and as a target for therapeutic intervention. Journal of Biological Chemistry 2016, 291(24):12538-12546.

25. Girlich D, Bernabeu S, Grosperrin V, Langlois I, Begasse C, Arangia N, Creton E, Cotellon G, Sauvadet A, Dortet L: Evaluation of the Amplidiag CarbaR+ MCR kit for the accurate detection of carbapenemaseproducing and colistin-resistant bacteria. Journal of clinical microbiology 2018:JCM. 01800-01818.

26. Kutter E, Sulakvelidze A: Bacteriophages: biology and applications: Crc press; 2004.

27. Merabishvili M, Vandenheuvel D, Kropinski AM, Mast J, De Vos D, Verbeken G, Noben J-P, Lavigne R, Vaneechoutte M, Pirnay J-P: Characterization of newly isolated lytic bacteriophages active against Acinetobacter baumannii. PloS one 2014, 9(8).

28. Lin N-T, Chiou P-Y, Chang K-C, Chen L-K, Lai M-J: Isolation and characterization of $\phi A B 2$ : a novel bacteriophage of Acinetobacter baumannii. Research in microbiology 2010, 161(4):308-314.

29. SADEGHIFARD NK, RANJBAR R, GHASEMI A, Pakzad I, ZAEIMI YJ, Zaheri A, HEMATIAN A, Ghafourian S: A study of antimicrobial resistance of acinetobacter baumannii and non-acinetobacter baumannii strains isolated from three hospitals in Tehran. 2006.

30. Sepahvand V, Davarpanah MA, Hejazi SH: Epidemiology of colistin-resistant Acinetobacter baumannii in Shiraz, Iran. J Appl Environ Biol Sci 2015, 5(5):45-48.

31. Dheepa M: Comparision of biofilm production and multiple drug resistance in clinical isolates of Acinetobacter baumanii from a tertiary care hospital in South India. 2011.

32. Rao RS, Karthika RU, Singh S, Shashikala P, Kanungo R, Jayachandran S, Prashanth K: Correlation between biofilm production and multiple drug resistance in imipenem resistant clinical isolates of Acinetobacter baumannii. Indian journal of medical microbiology 2008, 26(4):333.

33. Carson L, Gorman SP, Gilmore BF: The use of lytic bacteriophages in the prevention and eradication of biofilms of Proteus mirabilis and Escherichia coli. FEMS Immunology \& Medical Microbiology 2010, 59(3):447-455.

34. Mirzaei MK, Nilsson AS: Isolation of phages for phage therapy: a comparison of spot tests and efficiency of plating analyses for determination of host range and efficacy. PLoS One 2015, 10(3).

Figures 


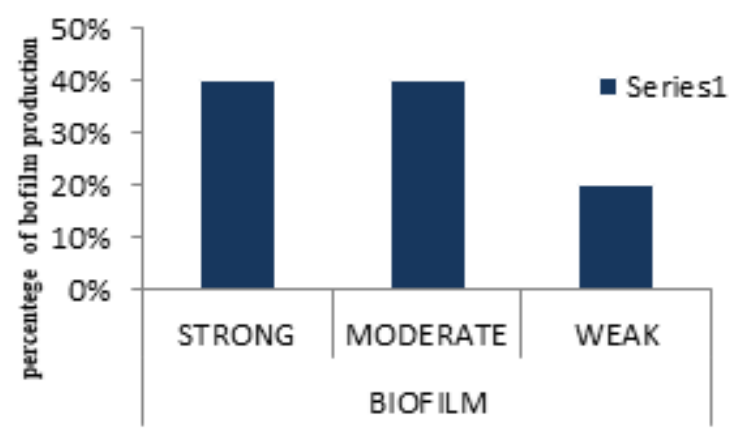

\section{Figure 1}

total isolates $(n=15)$; OD 570: biofilm formation was quantified by measuring optical absorbance ( 570 $\mathrm{nm}$ ) using crystal violet.

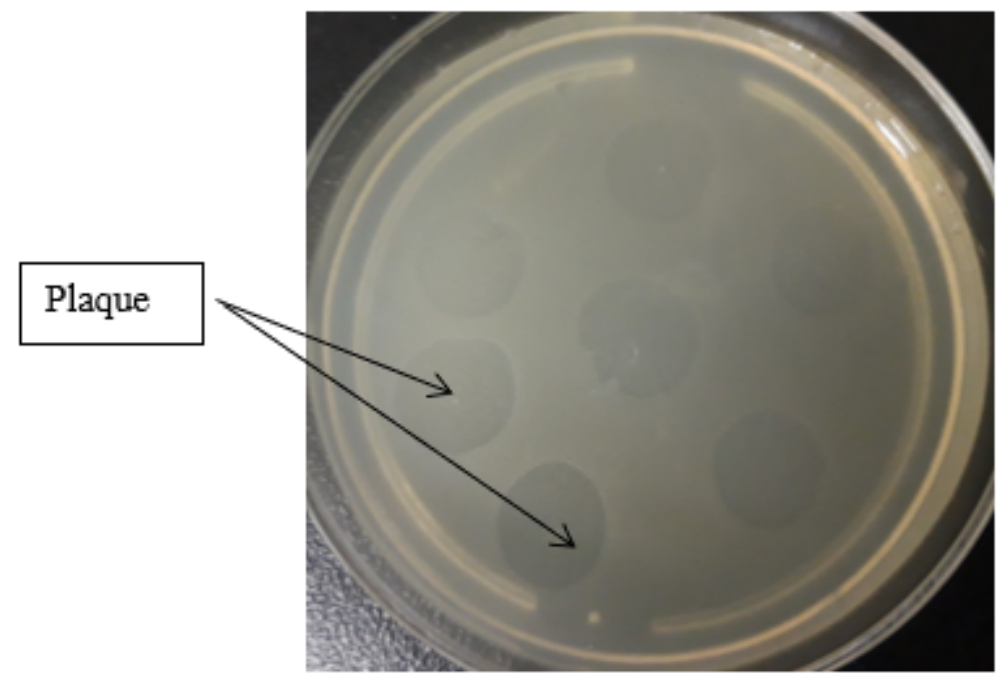

Figure 2

Spot assay for identification phage on the nutrient agar plate after $24 \mathrm{~h}$

\section{Supplementary Files}

This is a list of supplementary files associated with this preprint. Click to download.

- Supplementary.pdf 